\title{
Advanced Multilayer Composite Heavy-Oxide Scintillator Detectors for High Efficiency Fast Neutron Detection
}

\author{
Vladimir D. Ryzhikov, Member, IEEE, Sergei V. Naydenov*, Thierry Pochet, \\ Gennadiy M. Onyshchenko, Leonid A. Piven, and Craig F. Smith
}

\begin{abstract}
We have developed and evaluated a new approach to fast neutron and neutron-gamma detection based on largearea multilayer composite heterogeneous detection media consisting of dispersed granules of small-crystalline scintillators contained in a transparent organic (plastic) matrix. Layers of the composite material are alternated with layers of transparent plastic scintillator material serving as light guides. The resulting detection medium - designated as ZEBRA - serves as both an active neutron converter and a detection scintillator which is designed to detect both neutrons and gamma-quanta. The composite layers of the ZEBRA detector consist of small heavyoxide scintillators in the form of granules of crystalline BGO, GSO, ZWO, PWO and other materials. We have produced and tested the ZEBRA detector of sizes $100 \times 100 \times 41 \mathrm{~mm}$ and greater, and determined that they have very high efficiency of fast neutron detection (up to $49 \%$ or greater), comparable to that which can be achieved by large sized heavy-oxide single crystals of about $040 \times 80 \mathrm{~cm}^{3}$ volume. We have also studied the sensitivity variation to fast neutron detection by using different types of multilayer ZEBRA detectors of $100 \mathrm{~cm}^{2}$ surface area and $41 \mathrm{~mm}$ thickness (with a detector weight of about $1 \mathrm{~kg}$ ) and found it to be comparable to the sensitivity of a ${ }^{3} \mathrm{He}$-detector representing a total cross-section of about $2000 \mathrm{~cm}^{2}$ (with a weight of detector, including its plastic moderator, of about $120 \mathrm{~kg}$ ). The measured count rate in response to a fast neutron source of ${ }^{252} \mathrm{Cf}$ at $2 \mathrm{~m}$ for the ZEBRA-GSO detector of size $100 \times 100 \times 41 \mathrm{~mm}^{3}$ was $2.84 \mathrm{cps} / \mathrm{ng}$, and this count rate can be doubled by increasing the detector height (and area) up to $200 \times 100 \mathrm{~mm}^{2}$. In summary, the ZEBRA detectors represent a new type of high efficiency and low cost solid-state neutron detector that can be used for stationary neutron/gamma portals. They may represent an interesting alternative to expensive, bulky gas counters based on ${ }^{3} \mathrm{He}$ or ${ }^{10} \mathrm{~B}$ neutron detection technologies.
\end{abstract}

Index Terms-Fast Neutrons, Neutron/Gamma ray detection, Scintillator detectors, Nuclear Electronics, Nuclear Security

Manuscript received July 15, 2017. This work was supported in part by the NATO Science for Peace Program (Project SfP-984605)

V. D. Ryzhikov is with Institute for Scintillation Materials (ISMA) of the National Academy of Sciences of Ukraine, 61001 Kharkov, Ukraine (e-mail: ryzhikov@isma.kharkov.ua).

*S. V. Naydenov is with Institute for Single Crystals (ISC) of the National Academy of Sciences of Ukraine, 61001 Kharkov, Ukraine (e-mail: sergei.naydenov@gmail.com) (Corresponding Author).

T. Pochet is with International Atomic Energy Agency (IAEA), 1400 Vienna, Austria (e-mail: tpochet@detecrad.com).

G. M. Onyshchenko, L. A. Piven are with Institute for Scintillation Materials of the National Academy of Sciences of Ukraine, Kharkov, Ukraine.

C. F. Smith is with Naval Postgraduate School, Monterey, CA, USA (email: craigfsmith@yahoo.com).

\section{INTRODUCTION}

$\mathrm{O}$ ne of the main challenges in nuclear security is the creation of detection systems for fast neutrons and mixed neutron/gamma radiation that would be highly efficient, smallsized, inexpensive, easy-to-use, rugged and robust with respect to unfavorable conditions, and reliable in operation. Reliable recognition of illegal nuclear materials, in particular, weapon-grade uranium U-235 and plutonium Pu-239, as well as certain radioactive constituents of "dirty" bombs, requires the creation of detectors with high detection efficiency for fast neutrons (not less than 50\%). At the same time, practically all the newly-proposed detectors for the replacement of He-3 counters can efficiently detect only slow/thermal neutrons, relying on the radiative capture reactions of Li-6 or B-10 nuclei with very high thermal absorption cross-sections, but relative insensitivity to fast neutrons. The probability of direct detection of fast neutrons with these Li-6 or B-10 systems without using plastic moderators is orders of magnitude lower and, the efficiency of direct detection therefor does not exceed a few per cent. Even with massive and voluminous moderators to thermalize the fast neutrons, the fast neutron detection efficiency is not higher than $10-20 \%$, and for He-3 detectors about $10 \%$.

At the same time, we developed a new approach to neutron detector design, using single crystals of heavy-oxide scintillators (CWO, ZWO, PWO, BGO, GSO(Ce), etc.) for direct detection of fast neutrons without large scale thermalization [1-8]. This allowed us to realize increased fast neutron detection efficiencies of up to $50-70 \%$. For detection of fast neutrons, the inelastic scattering reaction on heavy nuclei was used, and the scintillator single crystal played a double role (two-in-one) - as an efficient converter of neutrons into secondary gamma-quanta of intermediate energies, and as a scintillator that detects these internal gamma-quanta in either a counting or spectrometric mode.

Our further studies have shown the possibility to also create a comparably efficient detector of fast neutrons on the basis of a novel composite scintillation structure, again relying on direct detection of fast neutrons through their interaction with materials of high effective atomic number. The fabrication costs of such composite scintillator structures can be 5-10 times lower in comparison with single crystals of comparable 
size. As a basic element, we developed an original heterogeneous scintillation structure (we use the name ZEBRA for it), which is comprised of a series of alternating plates of two types - plates consisting of small particles of heavy crystalline scintillator material (i.e., BGO, ZWO, PWO, $\mathrm{GSO}(\mathrm{Ce})$, etc.) embedded in a plastic scintillator matrix, and plates of a clear plastic moderator material, which simultaneously serves as a light guide for output of scintillation photons. In detecting fast neutrons, such a detector medium utilizes, to the maximum extent, not just one, but several channels of neutron interaction with heavy nuclei inelastic scattering of fast neutrons scattered at different angles into the moderator, as well as resonance scattering and radiative capture of neutrons moderated inside the detector by elastic scattering in hydrogen-containing plastic. For the moderator/light guide layers, one can also use plates of plastic scintillators which serve the additional functions of scintillation and spectrum-shifting activation. (In the latter case, the layer of plastic serves also as scintillator that simultaneously detects both gamma-quanta emitted in the heavy oxide scintillator layer as a result of inelastic and resonance scattering of fast neutrons, and recoil protons from elastic scattering of fast neutrons in the plastic itself). The detection of fast neutrons simultaneously over several channels of their interaction substantially increases total detection efficiency.

\section{EXPERIMENTAL SETUP FOR NEUTRON MEASUREMENTS}

We report on the research and development of fast neutron detectors based on large-size solid-state single crystal as well as multi-layer composite heavy-oxides $\mathrm{CWO}, \mathrm{ZWO}, \mathrm{BGO}$ and $\mathrm{GSO}(\mathrm{Ce})$ scintillators. The experimental setup and scheme of the experiments are shown on Figs. 1-2. Detection efficiency measurements for fast neutrons from a ${ }^{239} \mathrm{Pu}-\mathrm{Be}$ source by different scintillator detectors were made using a scintillation gamma-spectrometer with an analog-to-digital converter (ADC), which allowed recording of pulses from $1 \mu$ s up to $30 \mu \mathrm{s}$. The double integration time of the spectrometer amplifier was $30 \mu \mathrm{s}$, and the voltage on the PMT was $680 \mathrm{~V}$. The spectrometer was equipped with a charge-sensitive preamplifier. To obtain unambiguous correspondence between the occurrence of a neutron interaction and the appearance of the counting pulse in the working window of the detection system, the spectrometric signal integration time constant was chosen to be sufficiently high for all scintillators.

From the amplifier-shaper output, the signals come to the ADC input in the gamma-quanta energy range of $20-300 \mathrm{keV}$. The measured value was the number of spectrometer pulses in the working spectrometric window $(20-300 \mathrm{keV})$ during the same time interval.

Our method of measuring neutron detection efficiency assumes a "spherical" geometry and isotropic nature of the neutron source. Because of its low ambiguity, repeat measurements did not vary more than $1 \%$ with respect to the measured neutron flux.

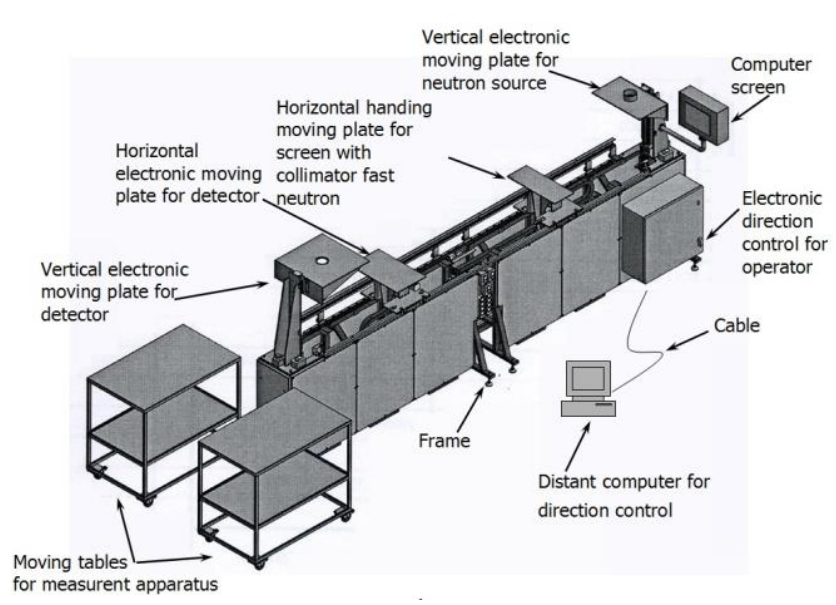

Fig. 1. Design of the experimental setup for fast neutron measurements. Length of hardware system $-3 \mathrm{~m}$. Height $-1.5 \mathrm{~m}$. Position accuracy of electronic-controlled plate $-0.1 \mathrm{~cm}$.

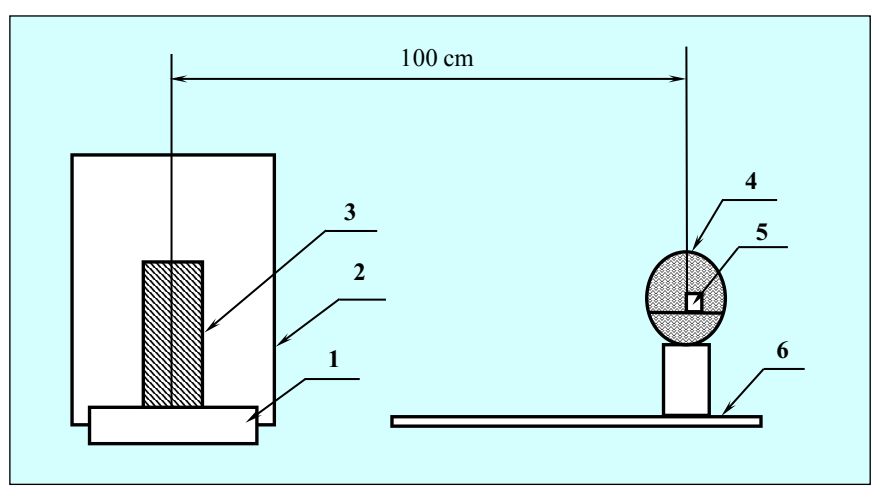

Fig. 2. Scheme of the experiments for fast neutron measurement: $1-$ PMT; 2 - enclosure to exclude external light; 3 - detector; 4 - ball $(\mathrm{R}=5 \mathrm{~cm})$ with hole for shielding of $\gamma$-radiation; 5 - neutron source ${ }^{239} \mathrm{Pu}-\mathrm{Be}$ or ${ }^{252} \mathrm{Cf} ; 6-$ foundation base.

\section{Multi-Layer Composite HeAvy-OXIDE SCINTILlator DETECTORS}

To detect radiation (i.e., neutron/gamma flux), we prepared a new form of composite scintillator fast neutron detectors (brand ZEBRA). These detectors are comprised of layers of a composite of granular scintillator particles embedded in transparent plastic (the dimension of the granules is about $0.2 \mathrm{~mm}$, and the layer thickness of $5 \mathrm{~mm}$ ). These composite layers are alternated with layers of plastic light guide/ scintillator material of thickness $5 \mathrm{~mm}$. In the light channel layers, light is collected and transmitted to the edge of the guide and then registered by a PMT (Hamamatsu model R1307).

The assembly of the 13-layer detectors $\left(100 \times 100 \times 41 \mathrm{~mm}^{3}\right)$ was performed by optical coupling of the single-layer scintillation panels ( $1 \mathrm{~mm}$ thicknesses) with light guides (5 $\mathrm{mm}$ thicknesses) with all surfaces polished. All of these detectors were carefully studied, and their neutron registration effectiveness was preliminarily tested using ${ }^{239} \mathrm{Pu}-\mathrm{Be}$ and ${ }^{252} \mathrm{Cf}$ neutron-gamma sources. The sketch on the Fig. 3 and photo 
on Fig. 4 illustrate the detector medium and its scheme of operation with neutron radiation and its registration.

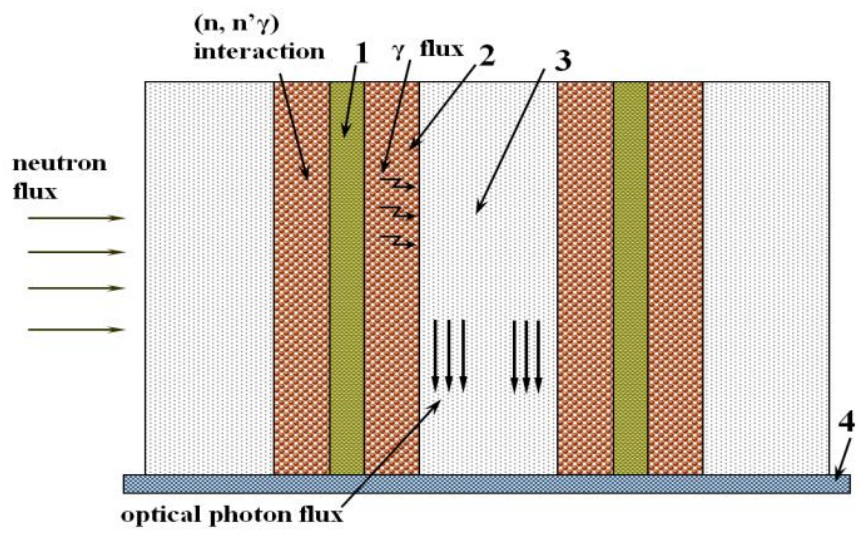

Fig. 3. Sketch of the design and operation of ZEBRA-brand multi-layer composite scintillator detectors.

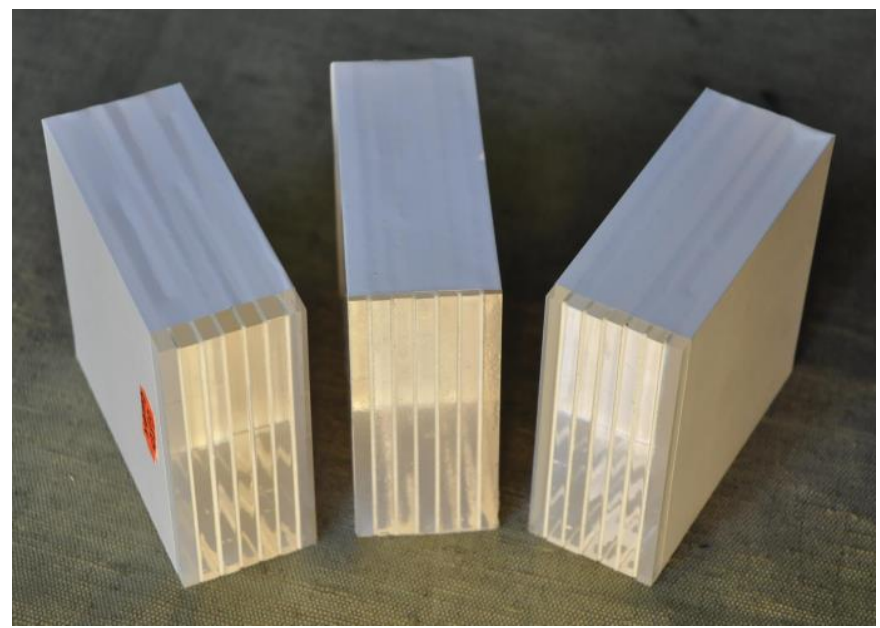

Fig. 4. Photo of ZEBRA-detectors with different composite scintillators $\mathrm{GSO}(\mathrm{Ce}), \mathrm{BGO}$ and ZWO. The size of every detector is $100 \times 100 \times 41 \mathrm{~mm}^{3}$.

Our measurements of the dependence of detection efficiency of single crystal detectors on crystal thickness show a practically linear relationship up to $40 \mathrm{~mm}$ [4]. But for the composite multilayer ZEBRA detectors with 5-6 composite layers of thickness 1-2 mm, i.e., a total thickness of heavyoxide scintillating layers of about $10 \mathrm{~mm}$, we find that the ZEBRA detector sensitivity is equal to that of single crystals of diameter of $40 \mathrm{~mm}$ and height of about $80 \mathrm{~mm}$ or more.

We suspect that this is due to the presence of the additional plastic in the composite layers and in the alternate layers of light guide. We believe that the interaction of neutrons with these materials include resonance and radiative capture interactions with much higher interaction cross sections. The process of neutron scattering within the detector can result in a decrease of neutron energy below the inelastic threshold. For this reason, in addition to detecting the signal from inelastic scattering, our method includes measurements in other channels to also detect neutrons of reduced energy which interact by resonance scattering and thermal capture (Fig. 5).

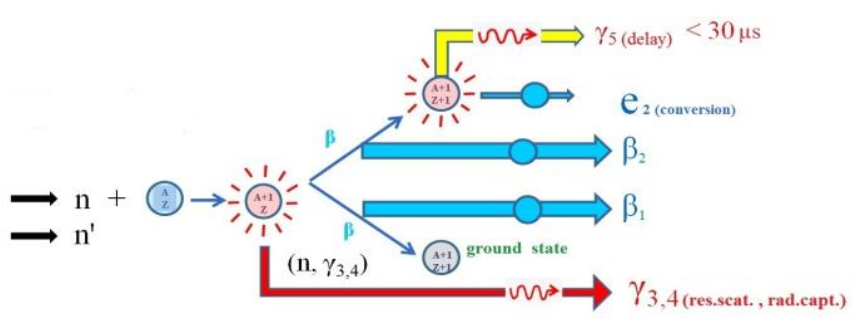

Fig. 5. The principal scheme of sources (physical channels) of registration signals from decelerated neutrons within the scintillator medium including (1) prompt gammas from resonant (non-elastic) neutron scattering and radiative capture as well as (2) beta-particles and internal-conversion electrons following such captures.

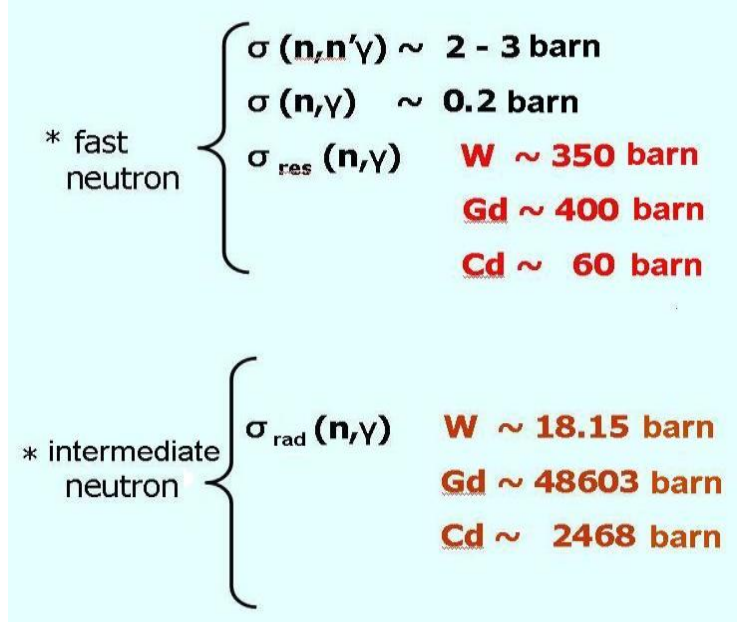

Fig. 6. Specific mechanisms of non-elastic scattering of neutrons with inelastic scattering, resonance and thermal capture cross sections for a $\mathrm{Pu}-\mathrm{Be}$ source (average fast neutron energy $\sim 4 \mathrm{MeV}$ ) for native "heavy" nuclei $\mathrm{W}$, $\mathrm{Gd}$ and $\mathrm{Cd}$ contained in heavy-oxide scintillators CWO, ZWO, GSO(Ce).

We observe a significant increase (up to $70-80 \%$ ) in the efficiency of fast neutron detection by single crystal detectors with the use of a surrounding layer of plastic of thickness from 1 to $10 \mathrm{~mm}$. This can be explained as a shift of neutron energy due to moderation in and below the resonance region. Registration of this interaction is enhanced by the fact that the cross-section for radiative capture $\sigma(\mathrm{n}, \gamma)$ is much greater than that of inelastic scattering $\sigma\left(n, n^{\prime} \gamma\right)$ as it is clearly presented on Fig. 6.

\section{FAST NEUtron Detectors EFFICIENCY AND PERFORMANCE}

Having prepared samples of this multi-layer composite detection medium using different heavy oxide scintillator materials, we tested them and compared their performance with that of a crystalline detector and a ${ }^{3} \mathrm{He}$ systems.

The preliminarily measured detection efficiency for a ZEBRA-GSO(Ce) structure of dimension 100x100x41 $\mathrm{mm}^{3}$ (containing 6 active layers of the composite scintillator) is $49 \%$ and can be increased further by increasing its thickness, that is, by increasing the number of active layers. The detector sensitivity reaches $49 \mathrm{cps} /\left(\right.$ neutron $\cdot \mathrm{s}^{-1} \cdot \mathrm{cm}^{2}$ ), which corresponds to a sensitivity of $2.84 \mathrm{cps} / \mathrm{ng}$ when the neutron 
flux comes from a Cf-252 source at a distance of $2 \mathrm{~m}$ from the detector. Thus, the obtained sensitivity exceeds the generally accepted standard of $2.5 \mathrm{cps} / \mathrm{ng}$. Moreover, the sensitivity of ZEBRA detectors can be made twice as high - up to $5 \mathrm{cps} / \mathrm{ng}$ and more - by increasing the area of the plates.

Fig. 7 shows the results of this comparative assessment and also indicates the measured efficiency of the different heavyoxide scintillators in crystalline and composite forms.

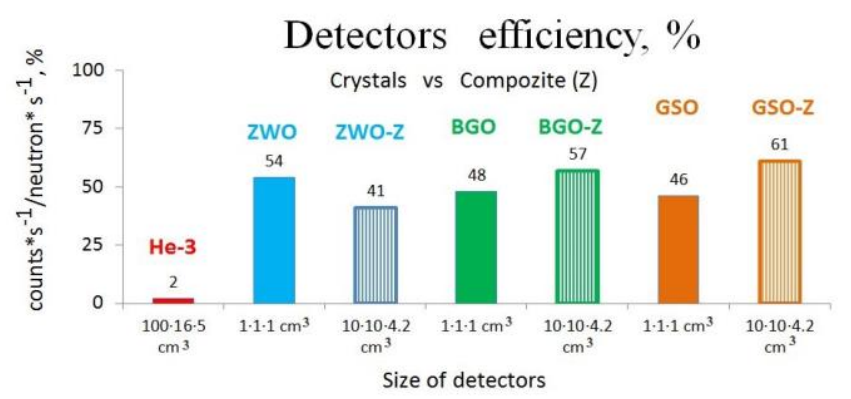

Fig. 7. Comparison of the fast neutron detection efficiency for single crystals and ZEBRA-brand multi-layer composite scintillator detectors.

The efficiency and sensitivity of neutron detectors are improved by increasing the cross sectional area of the crystal across the direction of incident neutron flux. To increase neutron detection sensitivity, theoretical and experimental investigations clearly indicate that the cross-sectional area (as well as the volume) of the detector facing the direction of neutron flux should be increased, with the desired crosssectional area being up to tens or hundreds of square centimeters. We can accomplish this objective namely by creating of large-volume multi-layer detectors in which a composite of scintillators containing active granules is placed inside a transparent polymer material. Because of the relatively low transparency of the composite inorganic scintillator, better light collection conditions are ensured by incorporation of a light guide between the composite scintillator layers. The design and fabrication technology of this detector allows the creation of neutron-gamma detectors characterized by high sensitivity at relatively low costs (as compared with analogs using oxide scintillators) for portable inspection systems. The sandwich structure can be comprised of any number of plates, with no limitations for thickness or area.

At the same time, to investigate the possibility of suppressing accompanying gamma-radiation, we have also developed a method for detection of fast neutrons against the background of intense gamma-radiation. Neutron-gamma separation was achieved using a specially developed algorithm based on measured data by using the well-known "window" method for different parts of the energy spectrum. This will permit us to achieve a $n / \gamma$ ratio rejection of $10^{4}-10^{5}$ and more.

\section{CONCLUSION}

We created and characterized a new type of multilayer composite detector (brand ZEBRA). In this detector medium, a composite that is comprised of granules of the scintillators
BGO, ZWO, GSO dispersed in transparent plastic is alternated with layers of clear plastic that serve as light guides. The sensitivity of these detectors with a size of 100x100x41 $\mathrm{mm}^{3}$ compares favorably with detectors based on large size crystals, but the composite structures are much cheaper and capable of manufacture in much larger dimensions.

This work represents a significant advancement from earlier single-crystal detector types as part of our effort to explore alternatives and improvements to the conventional ${ }^{3} \mathrm{He}$ counter. The current work directly detects fast neutrons while the ${ }^{3} \mathrm{He}$ counter relies of neutron thermalization prior to detection. This limitation of the ${ }^{3} \mathrm{He}$ counter restricts its efficiency in fast neutron detection to not more than about $10 \%$, whereas the new method of direct detection of fast neutrons enables efficiencies of up to $50-60 \%$.

The anomalously high efficiency of the composite scintillators can be only qualitatively explained by assuming of interaction of neutrons with the scintillator. More detailed theoretical analysis would be desirable, with quantitative calculations for these mechanisms. Further development of the composite detector design and choice of optimum detector materials need for to ensure the best "price-quality" ratio would be also necessary.

\section{ACKNOWLEDGMENT}

This research is supported in part by NATO's Emerging Security Challenges Division in the Science for Peace and Security Program under Project SfP-984605.

\section{REFERENCES}

[1]V.D. Ryzhikov, B.V. Grinyov, G.M. Onyshchenko, L.A. Piven, O.K. Lysetska, L.L. Nagornaya, T. Pochet, "The use of fast and thermal neutron detectors based on oxide scintillators in inspection systems for prevention of illegal transportation of radioactive substances", IEEE Trans. Nucl. Sci, vol. 57, No. 5, pp. 2747, 2010.

[2]V.D. Ryzhikov, B.V. Grinyov, G.M. Onyshchenko, L.A. Piven, O.K. Lysetska, O.D. Opolonin, S.A. Kostioukevitch, C.F. Smith, "The neutron detectors based on oxide scintillators for control of fissionable radioactive substances", in Proc. of SPIE, Hard X-Ray, Gamma-Ray, and Neutron Detector Physics XVI, vol. 92131B, September 5, 2014; doi:10.1117/12.2058185.

[3]V.D. Ryzhikov, S.V. Naydenov, G.M. Onyshchenko, T. Pochet, C.F. Smith, "High efficiency fast neutron detectors based on inorganic scintillators", in IEEE 2014 Nuclear Science Symposium, Seattle, WA, USA, 2014; doi:10.1109/NSSMIC.2014.7431165.

[4]V. Ryzhikov, C. Smith, B. Grinyov, L. Piven, G. Onyshchenko, S. Naydenov, T. Pochet, "Detection of gamma-neutron radiation by novel solid-state scintillation detectors", in Proceeding of ANIMMA-2015, Lisbon, Portugal, 2015; doi:10.1109/ANIMMA.2015.7465541.

[5]B. Grinyov, V. Ryzhikov, L. Nagorna, G. Onyshchenko, L. Piven, "Smallsized detector of fast and thermal neutrons", Patent of Ukraine \#92193, Oct. 11, 2010.

[6]B. Grinyov, V. Ryzhikov, L. Nagornaa, G. Onyshchenko, L. Piven, "Method of registration of fast neutrons", Patent of Ukraine \#96428, Nov. 11, 2011.

[7]B. Grinyov, V. Ryzhikov, L. Nagornaa, G. Onyshchenko, L. Piven "Method of detection of fast neutrons", Patent of USA No. US 8058624 B2, Nov. 15, 2011.

[8]V. Ryzhikov, B. Grinyov, S. Naydenov, G. Onyshchenko, L. Piven, "Method of registration of fast neutrons and combined detector based thereon", Patent Application of Ukraine \#a201611057, Nov. 11, 2016. 